Bang-yen Chen

Nagoya Math. J.

Vol. 60 (1976), 1-6

\title{
SOME RELATIONS BETWEEN DIFFERENTIAL GEOMETRIC INVARIANTS AND TOPOLOGICAL INVARIANTS OF SUBMANIFOLDS ${ }^{1}$
}

\author{
BANG-YEN CHEN ${ }^{2)}$
}

\section{§ 1. Introduction.}

Let $M$ be an $n$-dimensional manifold immersed in an $m$-dimensional euclidean space $E^{m}$ and let $\nabla$ and $\tilde{\nabla}$ be the covariant differentiations of $M$ and $E^{m}$, respectively. Let $X$ and $Y$ be two tangent vector fields on $M$. Then the second fundamental form $h$ is given by

$$
\tilde{\nabla}_{X} Y=\nabla_{X} Y+h(X, Y) \text {. }
$$

It is well-known that $h(X, Y)$ is a normal vector field on $M$ and it is symmetric on $X$ and $Y$. Let $\xi$ be a normal vector field on $M$, we write

$$
\tilde{\nabla}_{X} \xi=-A_{\xi}(X)+D_{X} \xi,
$$

where $-A_{\xi}(X)$ and $D_{X \xi}$ denote the tangential and normal components of $\tilde{\nabla}_{X} \xi$. Then we have

$$
\left\langle A_{\xi}(X), Y\right\rangle=\langle h(X, Y), \xi\rangle,
$$

where $\langle$,$\rangle denotes the scalar product in E^{m}$. The mean curvature vector $H$ is defined by $H=(1 / n)$ trace $h$. Let $S$ denote the length of $h$ and $\alpha$ the length of $H$.

In this paper we shall obtain some relations between differential geometric invariants and a topological invariants of $M$. In particular, we shall prove that, for any closed $n$-dimensional submanifold $M$ in $E^{m}$, the geometric invariant given by the integral of $S^{n}$ depends on a topol-

Received July 22, 1974.

Revised September 18, 1975.

1) A partial result of this paper was announced in the following article "Some integral inequalities of two geometric invariants" appeared in Bull. Amer. Math. Soc. 81 (1975), 177-178.

2) This work was partially supported by NSF Grant GP-36684. 
ogical structure of $M$. Moreover, if the submanifold is $\delta$-pinching in $E^{m}$ (for the definition, see $\S 4$ ), then the total mean curvature, i.e., the geometric invariant given by the integral of $\alpha^{n}$, also depends on the same topological structure of $M$. In particular, we see that among all $\delta$-pinching submanifolds in $E^{m}$ with a fixed $\delta>-1$, the submanifolds with large homology groups must have large total mean curvature.

\section{§ 2. Basic formulas.}

Let $\xi$ be a unit normal vector field on $M$. We define the $i$-th mean curvature $K_{i}(\xi)$ at $\xi$ by

$$
\operatorname{det}\left(I+t A_{\xi}\right)=1+\sum_{i=1}^{n}\left(\begin{array}{c}
n \\
i
\end{array}\right) K_{i}(\xi) t^{i},
$$

where $I$ is the identity transformation of the tangent spaces of $M, t$ a parameter and $\left(\begin{array}{c}n \\ i\end{array}\right)=n ! / i !(n-i) !$. Let $R$ be the curvature tensor of $M$, i.e.,

$$
R(X, Y)=\nabla_{X} \nabla_{Y}-\nabla_{Y} \nabla_{X}-\nabla_{[X, Y]} \cdot
$$

Then the Gauss equation is given by

$$
\langle R(X, Y) Z, W\rangle=\langle h(Y, Z), h(X, W)\rangle-\langle h(X, Z), h(Y, W)\rangle .
$$

Let $E_{1}, \cdots, E_{n}$ be local orthonormal tangent vector fields of $M$. Then the scalar curvature $\rho$ is defined by

$$
\rho=\sum_{j=1}^{n}\left(\sum_{i=1}^{n}\left\langle R\left(E_{i}, E_{j}\right) E_{j}, E_{i}\right\rangle\right) .
$$

From (2.2) and (2.3) we have

$$
\rho=n^{2} \alpha^{2}-S^{2} .
$$

\section{§ 3. Integral inequality for $S^{n}$.}

Let $\mathscr{F}$ be a field and let $H_{i}(M ; \mathscr{F})$ be the $i$-th homology group of $M$ over the field $\mathscr{F}$. Let $\beta_{i}(M ; \mathscr{F})$ be the dimension of the $i$-th homology group $H_{i}(M ; \mathscr{F})$. We define a topological invariant $\beta(M)$ by

$$
\beta(M)=\max \left\{\sum_{i=0}^{n} \beta_{i}(M ; \mathscr{F}): \mathscr{F} \text { fields }\right\} .
$$

The main aim of this section is to prove the following. 
THEOREM 1. Let $M$ be an n-dimensional closed manifold immersed in a euclidean $m$-space $E^{m}$. Then we have

$$
\int_{M} S^{n} d V \geqq\left\{\left(\frac{n}{2}\right)^{n / 2} c_{n}\right\} \beta(M),
$$

where $c_{n}$ is the area of a unit $n$-sphere. The equality sign of (3.1) holds when and only when $M$ is diffeomorphic to an $n$-sphere and $M$ is imbedded as a hypersphere of an $(n+1)$-dimensional linear subspace of $E^{m}$.

Proof. Let $M$ be an $n$-dimensional closed manifold immersed in $E^{m}$ and $\xi$ be any unit normal vector field on $M$. We denote by $S(\xi)$ the length of the second fundamental tensor $A_{\xi}$ at $\xi$. Let $\xi_{1}, \cdots, \xi_{m-n}$ be local orthonormal normal vector fields of $M$ in $E^{m}$ and $\xi=\sum_{r=1}^{m-n} \cos \gamma_{r} \xi_{r}$. Then we have

$$
A_{\xi}=\sum \cos \gamma_{r} A_{r}, \quad A_{r}=A_{\xi r} .
$$

Hence we have

$$
S(\xi)^{2}=\operatorname{trace}\left(A_{\xi}^{2}\right)=\sum_{r, s} \cos \gamma_{r} \cos \gamma_{s} \operatorname{trace}\left(A_{r} A_{s}\right) .
$$

The right hand side of (3.3) is a quadratic form on $\cos \gamma_{1}, \cdots, \cos \gamma_{m-n}$. Hence, we may choose local orthonormal normal vector fields $\bar{\xi}_{1}, \ldots, \bar{\xi}_{m-n}$ such that with respect to this frame field, we have

$$
\begin{gathered}
S(\xi)^{2}=\sum \rho_{r} \cos ^{2} \gamma_{r}, \quad \rho_{1} \geqq \rho_{2} \geqq \cdots \geqq \rho_{m-n} \geqq 0, \\
\rho_{r}=\operatorname{trace}\left(A_{r}^{2}\right)=S\left(\xi_{r}\right)^{2} .
\end{gathered}
$$

By the definition of $S$ and $\rho_{r}$ we have

$$
S^{2}=\rho_{1}+\cdots+\rho_{m-n} .
$$

In the following, let $B_{\nu}$ be the bundle of unit normal vectors of $M$ in $E^{m}$ so that a point of $B_{\nu}$ is a pair $(x, \xi)$ where $\xi$ is a unit normal vector at the point $x$ in $M$. Then $B_{\nu}$ is a bundle of $(m-n-1)$-dimensional spheres over $M$ and is a manifold of dimension $m-1$. Let $\Sigma_{x}$ be the fibre of $B_{\nu}$ over $x$. Then there is a differential form $d \sigma$ of degree $m-n-1$ on $B_{v}$ such that its restriction to a fibre $\Sigma_{x}$ is the volume element $d \Sigma_{x}$ of $\Sigma_{x}$. Hence $d \sigma \wedge d V$ is the volume element of the bundle $B_{\nu}$. On the bundle $B_{\nu}$ we define a function $f$ by

$$
f(x, \xi)=S(\xi)^{2} \text {. }
$$


For $\xi=\sum \cos \gamma_{r} \bar{\xi}_{r}$ we have

$$
f(x, \xi)=\sum \rho_{r} \cos ^{2} \gamma_{r}
$$

Since $\rho_{r}, r=1, \cdots, m-n$, are nonnegative and $\sum_{r} \cos ^{2} \gamma_{r}=1$, an inequality of Minkowski [1, p. 21] implies that

$$
\begin{aligned}
& \left\{\int_{\Sigma_{x}} f^{n / 2} d \Sigma_{x}\right\}^{2 / n}=\left\{\int_{\Sigma_{x}}\left(\sum \rho_{r} \cos ^{2} \gamma_{r}\right)^{n / 2} d \Sigma_{x}\right\}^{2 / n} \\
& \quad \leqq \sum\left\{\rho_{r}\left(\int_{\Sigma_{x}}\left|\cos ^{n} \gamma_{r}\right| d \Sigma_{x}\right)^{2 / n}\right\}
\end{aligned}
$$

Moreover, we have the following identity:

$$
\int_{\Sigma_{x}}\left|\cos ^{n} \gamma_{r}\right| d \Sigma_{x}=2 c_{n+p-1} / c_{n}
$$

Thus, by combining (3.6), (3.9) and (3.10), we find

$$
S^{n} \geqq \frac{c_{n}}{2 c_{m-1}} \int_{\Sigma_{x}} f^{n / 2} d \Sigma_{x}
$$

On the other hand, from the definition of $K_{n}(\xi)$ and an elementary relation between elementary symmetric functions, we have $S(\xi)^{n} \geqq$ $\sqrt{n^{n}}\left|K_{n}(\xi)\right|$. Hence, by using (3.11), we see that

$$
\int_{M} S^{n} d V \geqq \sqrt{n^{n}} \frac{c_{n}}{2 c_{m-1}} \int_{B_{\nu}}\left|K_{n}(\xi)\right| d \sigma \wedge d V
$$

By a well-known inequality of Chern-Lashof $[4$, II], we have

$$
\int_{B_{\nu}}\left|K_{n}(\xi)\right| d \sigma \wedge d V \geqq c_{m-1} \beta(M) .
$$

Thus, by combining (3.12) and (3.13), we obtain (3.1).

The remaining part of this theorem can be proved in a similar way as the corresponding results of Theorem 4.2 in [2, p. 229]. So we omit it.

Remark 1. Theorem 1 generalizes Theorem 4.1 of [3, II]. First, Theorem 1 drops the assumption of nonnegativeness of the scalar curvature of $M$. Second, if $n$ is odd, the estimation is better than the one given in Theorem 4.1 of [3, II]. 


\section{§ 4. Total mean curvature.}

From Proposition 2.2 of [3, II] we see that the scalar curvature $\rho$ is always bounded from above by $(n-1) S^{2}$ and bounded below by $-S^{2}$, i.e.,

$$
-S^{2} \leqq \rho \leqq(n-1) S^{2}
$$

In the following, a submanifold $M$ in $E^{m}$ is said to satisfy a $\delta$-pinching in $E^{m}$ if we have

$$
\delta S^{2} \leqq \rho \leqq(n-1) S^{2}
$$

for some $\delta \geqq-1$.

THEOREM 2. Let $M$ be an n-dimensional closed manifold immersed in a euclidean $m$-space $E^{m}$. If $M$ satisfies a $\delta$-pinching in $E^{m}$, then we have

$$
\int_{M} \alpha^{n} d V \geqq\left\{\frac{1}{2}\left(\frac{1+\delta}{n}\right)^{n / 2} c_{n}\right\} \beta(M)
$$

The equality sign of (4.2) holds when and only when $M$ is $(n-1)$-pinching in $E^{m}$.

Proof. If $M$ is $\delta$-pinching in $E^{m}$, then (2.4) implies

$$
\alpha^{2} \geqq \frac{1+\delta}{2} S^{2}
$$

Hence, by combining Theorem 1 and (4.3) we obtain (4.2).

Now, if the equality sign of (4.2) holds, then the equality sign of (3.1) holds. Hence, Theorem 1 implies that $M$ is imbedded as a hypersphere of an $(n+1)$-dimensional linear subspace of $E^{m}$. In this case we have $n^{2} \alpha^{2}=n S^{2}$. Hence, by (2.4), we see that $M$ is $(n-1)$-pinching in $E^{m}$. The remaining part of this Theorem is trivial.

Remark 2. If $\delta>-1$ and $M$ is a minimal submanifold of a unit hypersphere of $E^{m}$, then $M$ is $\delta$-pinching in $E^{m}$ when and only when the scalar curvature $\rho$ of $M$ satisfies the following inequality:

$$
\rho \geqq \frac{\delta}{1+\delta} n^{2}
$$

In this case, $\int_{M} \alpha^{n} d V$ is equal to the volume of $M$. 


\section{REFERENCES}

[1] E. F. Beckenbach and R. Bellman, Inequalities, Springer-Verlag, Berlin, 1961.

[2] B.-Y. Chen, Geometry of Submanifolds, M. Dekker, New York, 1973.

[ 3 ] B.-Y. Chen, On the total curvature of immersed manifolds, I, Amer. J. Math. 93 (1971), 148-162; — II, Amer. J. Math. 94 (1972), 799-809; —, III, Amer. J. Math. 95 (1973), 636-642.

[4] S. S. Chern and R. K. Lashof, On the total curvature of immersed manifolds, I, Amer. J. Math. 79 (1957), 306-318; — II, Michigan Math. J. 5 (1958), 5-12.

Michigan State University 